\title{
Demografischer Wandel in der Erwerbsarbeit - Risiken und Potentiale alternder Belegschaften
}

\author{
Frerich Frerichs
}

Online publiziert: 14. November 2014

(C) Institut für Arbeitsmarkt- und Berufsforschung 2014

Zusammenfassung Der Beitrag analysiert vor dem Hintergrund des demografischen Wandels in der Erwerbsarbeit, welche Herausforderungen sich für eine produktive Nutzung alternder Belegschaften und die Förderung der Arbeitsfähigkeit älterer Arbeitnehmer im Betrieb stellen. Hierzu wird vor allem der Einfluss der Arbeitsbedingungen auf die Entwicklung der altersspezifischen Leistungsfähigkeit in den drei zentralen Feldern der Gesundheit, der Qualifikation und der Motivation herausgearbeitet. Die gegebenen Risiken und Chancen in der Entwicklung der Leistungsfähigkeit werden erklärend eingebunden in betriebliche Strategien der Humanressourcennutzung im Sinne von Produktionsregimen. Als handlungsleitend für eine Verbesserung der Leistungsfähigkeit wird ein System des Alternsmanagements dargestellt, das zum einen erwerbsbiografisch ausgerichtet ist und zum anderen eine integrative Betrachtungsweise der zentralen Handlungsfelder in der Arbeits- und Laufbahngestaltung, der Gesundheitsförderung und der betrieblichen Weiterbildung anstrebt. Im Ausblick wird deutlich gemacht, dass zur Umsetzung dieses Handlungsansatzes nicht nur die betrieblichen Rahmenbedingungen stärker berücksichtigt werden müssen, sondern auch die akteursbezogene Perspektive - z. B. in Form tarifvertraglicher Aushandlungsprozesse - gestärkt werden muss.

Prof. Dr. F. Frerichs $(\bowtie)$

Fachgebietsleiter „Altern und Arbeit“,

Institut für Gerontologie,

Universität Vechta,

Driverstr. 23,

49377 Vechta, Deutschland

E-Mail: frerich.frerichs@uni-vechta.de
Demographic change in gainful employment: risks and potentials of ageing workforces

\begin{abstract}
At the background of demographic change in gainful employment the paper analyses which challenges are faced to employ an ageing workforce productively and to foster the workability of older workers.

In particular, the influence of working conditions on the age-specific workability in the spheres of health, skills and motivation is stressed. The given risks and potentials in the development of workability are embedded in a clarifying system of human resource strategies on company level in the sense of production regimes. A system of age-management is enfolded which incorporates a longitudinal perspective and tries to integrate the relevant fields of action such as work design, career management, health promotion and training. Finally, it is stressed that for implementing this strategy successfully it is not only necessary to reflect the specific company frameworks but also to observe a stronger actor centered perspective, e.g. via collective agreements.
\end{abstract}

\section{Einführung}

Der demografische Wandel in der Erwerbsarbeit gewinnt eine weiter wachsende Bedeutung in der arbeits(markt) politischen und arbeitswissenschaftlichen Diskussion in Deutschland. Der Grund hierfür ist zum einen, dass die im Zusammenhang mit den Konsolidierungsbestrebungen der Rentenfinanzen vorgenommenen einschneidenden Veränderungen bei der Gestaltung des Rentenzugangs, die insgesamt auf eine Verlängerung der Lebensarbeitszeit abzielen, zunehmend an Gestalt gewinnen. Auch die - bereits seit Anfang der 90er Jahre in diesbezüglichen Prognosen her- 
ausgestellte - Alterung des Erwerbspersonenpotentials schlägt immer deutlicher auf die Betriebe durch und verstärkt den Trend hin zu alternden Belegschaften. Schließlich tragen die dynamischen Entwicklungen in der Arbeitslandschaft als solcher - Stichworte bilden hier u. a. die Begriffe Globalisierung, Flexibilisierung, Rationalisierung und verstärkte Dienstleistungsorientierung - dazu bei, dass alternde Belegschaften zahlreichen betrieblichen Veränderungen und Herausforderungen ausgesetzt sind. In diesem Zusammenhang werden sowohl Fragen bezüglich der Leistungs- und Innovationsfähigkeit der jetzt und zukünftig älteren Arbeitnehmer aufgeworfen, als auch arbeitsmarkt- und personalpolitische Fragestellungen hinsichtlich ihrer Integration in die Erwerbsarbeit thematisiert. Der folgende Beitrag stellt zum einen empirische Evidenzen zu Risiken und Potentialen alternder Belegschaften auf der betrieblichen Ebene zur Diskussion und versucht zum anderen theoretisch-konzeptionelle Hinweise zur Einordnung dieser Phänomene und zu personalpolitischen Handlungsansätzen zu geben.

\section{Demografischer Wandel und Erwerbsbeteiligung im Alter}

Vorliegenden demografischen Prognosen zufolge wird das Erwerbspersonenpotential zwar erst mittel- bis langfristig deutlich sinken, die Alterung setzt aber schon sehr kurzfristig ein (vgl. Fuchs et al. 2011). Auch wenn sich die Strukturveränderungen nicht völlig in den tatsächlichen Altersstrukturen in den Betrieben widerspiegeln werden und nach Branchen und Regionen zu differenzieren ist (vgl. u. a. Leber et al. 2013), so ist doch davon auszugehen, dass auch auf einzelbetrieblicher Ebene kaum eine Abkoppelung von diesen altersstrukturellen Verschiebungen möglich sein wird (Sporket 2011). Künftig werden daher die Erschließung des Leistungspotentials von älteren Beschäftigten und ihre produktive Integration in den Betrieb weiter an Stellenwert gewinnen. Für die Zukunft von Wirtschaft und Arbeitsmarkt auf der einen Seite und für die beruflichen Perspektiven der älteren Arbeitskräfte auf der anderen Seite wird insbesondere entscheidend sein, ob eine alternde Erwerbsbevölkerung den Anforderungen des wirtschaftsstrukturellen und technologisch-organisatorischen Wandels entsprechen kann bzw. ob die erforderlichen Anpassungsmaßnahmen rechtzeitig entwickelt werden.

Bereits seit Ende der 90er Jahre ist eine zunehmende Erwerbsbeteiligung der älteren Bevölkerung zu konstatieren. Dieser Anstieg ist insbesondere in der Altersgruppe der 60- bis 64-Jährigen ausgeprägt und zeigt sich bei den Frauen noch stärker als bei den Männern. So hat in dieser Altersgruppe im Zeitraum zwischen 1991 und 2011 die Erwerbstätigenquote bei den Frauen um rd. 26 Prozentpunkte von knapp $10 \%$ auf rd. $36 \%$ zugenommen, bei den Männern um rd. 21 Prozentpunkte von rd. $31 \%$ auf rd. 52\%. ${ }^{1}$ Als Grund hierfür können insbesondere die seit Beginn der 90er Jahre sukzessiv herbeigeführte Anhebung der Altersgrenzen für den Rentenbezug, die Einführung von Rentenabschlägen bei einem vorgezogenen Übergang in den Ruhestand und die weiter steigende Erwerbsbereitschaft von Frauen angeführt werden (Bäcker et al. 2009). Mit dieser Anhebung der Altersgrenzen werden die vorgängig dominierenden arbeitsmarktpolitischen Entlastungsargumente und - dies sollte nicht vergessen werden - die ebenfalls eine Rolle spielenden humanisierungspolitischen Überlegungen bezogen auf einen vorgezogenen Renteneintritt bei stark belastenden Arbeitsbedingungen (vgl. dazu Heinze et al. 2011) durch rentenfinanzpolitische Zielsetzungen abgelöst.

Allerdings ist damit keineswegs sichergestellt, dass Arbeitnehmer in der Spätphase ihrer Erwerbstätigkeit vollumfänglich und dauerhaft in Erwerbsarbeit integriert sind. So ist zu berücksichtigen, dass die Erwerbstätigenquote von geringqualifizierten älteren Arbeitnehmern deutlich unter der von höherqualifizierten liegt (Mümken und Brussig 2012) und in den Altersjahrgängen ab 60 Jahre die Erwerbsbeteiligung trotz der Wachstumsraten nach wie vor stark unterdurchschnittlich ist (BA 2012). Zudem zeigt sich, dass in der Spätphase der Erwerbsarbeit eine Beschäftigung oft nur noch mit reduziertem Arbeitsumfang realisiert werden kann - insbesondere bei den älteren Frauen liegt der Anteil der Teilzeit- und geringfügig Beschäftigten sehr hoch (Wanger 2006; Bäcker et al. 2010). Viele Arbeitnehmer scheiden zudem vorzeitig auf Grund gesundheitlicher Einschränkungen aus dem Erwerbsleben aus. So lag etwa der Anteil der Erwerbsminderungsrenten an allen Rentenneuzugängen des Jahres 2011 bei rd. 20\% (vgl. Bäcker 2012).

Zum gegenwärtigen Zeitpunkt ist daher in vielen Bereichen der Arbeitswelt fraglich, ob ältere Arbeitnehmer tatsächlich bis zum Erreichen der Altersgrenzen im Betrieb verbleiben und dort auch produktiv tätig sein können. Die vor allem von Seiten der Betriebe erforderlichen personalpolitischen Rahmenbedingungen und auf altersübergreifende Qualifizierung, Gesundheitsförderung und Personalentwicklung bezogene Maßnahmen fehlen noch weitgehend und es bestehen weiterhin Beschäftigungsrisiken, die von den konkreten Arbeits- und Beschäftigungsbedingungen ausgehen. In diesem Zusammenhang sei auf zwei besonders markante, arbeitsbedingte altersspezifische Risikofaktoren hingewiesen. So haben ältere Arbeitnehmer - verglichen mit Jüngeren - sowohl ein höheres Krankheits- als auch ein höheres Qualifizierungsrisiko (Naegele 2004).

Beide Risiken unterliegen „Karriereverläufen“, d. h. entwickeln sich im Verlauf der Arbeits- und Berufsbiographie - nicht selten schleichend -, erreichen in der Spätphase des Erwerbslebens ihren Höhepunkt, treten hier häufig kumu-

\footnotetext{
${ }^{1}$ Erwerbstätigenquote auf Grundlage des Mikrozensus.
} 
lativ auf und prägen dann ganz entscheidend die höheren Beschäftigungsrisiken Älterer mit (Kistler 2009). Die Ausprägung dieser Risiken ist stark von den Entwicklungstrends in der Arbeitsgesellschaft beeinflusst. Der wachsende Druck, der in den Betrieben auf arbeitsorientierte Rationalisierung hinweist (vgl. hierzu im Überblick Heinze et al. 2011; Hentrich und Latniak 2013), wirkt sich zwangsläufig auch auf die demografisch wachsende Zahl älterer Beschäftigter aus.

\section{Leistungsfähigkeit und Beschäftigungsrisiken älterer Arbeitnehmer}

Der Zusammenhang zwischen Alter und Leistungsfähigkeit im Betrieb wird stets nur über die Rahmenbedingungen der konkreten Arbeitssituation vermittelt. $\mathrm{Zu}$ beachten sind allerdings die ausgeübten Tätigkeiten und die damit verbundenen Arbeitsbedingungen über den gesamten Erwerbsverlauf. Zum einen ist hierbei zu berücksichtigen, inwiefern sich Tätigkeiten und Arbeitsbedingungen direkt auf die Leistungsfähigkeit auswirken, zum anderen muss betrachtet werden, welche Bedeutung Aspekte eines altersspezifischen Leistungswandels bei der konkreten Arbeitstätigkeit gewinnen.

Die konkreten Arbeitsbedingungen und die Verweildauer über den Berufsverlauf können spezifische Beschäftigungschancen und -probleme generieren. Ausmaß und Zeitpunkt des Auftretens beruflicher Leistungsveränderungen sind in hohem Maße von den aus den jeweiligen Arbeitsbedingungen resultierenden psychischen und physischen Belastungen und Handlungsspielräumen abhängig. Eine langfristige Tätigkeit an einem Arbeitsplatz mit hoher Belastungsexposition und fehlenden Möglichkeiten für körperliches und kognitives Training kann demnach Alterungsprozesse beschleunigen (,,arbeitsinduziertes Voraltern“, vgl. Hacker 2004, S. 164), wohingegen umgekehrt gesundheits-, qualifikations- und motivationsförderliche Arbeitszusammenhänge Leistungsrückgänge verzögern können bzw. die Kompensation von Defiziten ermöglichen (vgl. z. B. Morschhäuser 2006).

Hinzu kommen aus gerontologischer Sicht Differenzen und Dynamiken der Entwicklung der beruflichen Leistungsfähigkeit in den individuellen Alterungsprozessen. Hierbei handelt es sich um nicht-lineare und multidimensionale psycho-physische Veränderungsprozesse, die sowohl Rückgänge (z. B. der Muskelkraft, der Beweglichkeit, des Reaktions- und Koordinationsvermögen und der Geschwindigkeit der Informationsaufnahme und -verarbeitung), Zuwächse (Erfahrungswissen und Geübtheit, Verantwortungsbewusstsein, soziale Kompetenzen, Organisations- und Planungsfähigkeiten) als auch Konstanzen (Langzeitgedächtnis,
Konzentrations- und Merkfähigkeit) umfassen können (vgl. im Überblick Naegele 2004; Lehr 2007; Sonntag 2012).

In diesem Zusammenhang ist darauf hinzuweisen, dass viele der zumeist im Rahmen von Laboruntersuchungen festgestellten, alterskorrelierten kognitiven und physiologischen Abbauprozesse in der beruflichen Praxis nicht oder nur geringfügig wirksam werden, z. B. weil in den jeweiligen Fähigkeitsbereichen nicht die maximale Leistung abgerufen werden muss oder einzelne Minderleistungen durch Leistungsstärken in anderen Funktionsbereichen bzw. einen veränderten Einsatz der vorhandenen Ressourcen kompensiert werden können. So kann Erfahrungswissen z. B. einen Ausgleich von Rückgängen bei Ausdauer und Schnelligkeit durch effizientere bzw. vorausschauendere Arbeitsweisen ermöglichen (Zimmermann 2005). Entsprechende Effekte führen dazu, dass in der Summe häufig keine oder nur geringfügige Leistungs- bzw. Produktivitätsrückgänge bei älteren Arbeitnehmern beobachtbar sind (vgl. aktuell hierzu Schlick et al. 2013).

Umgekehrt gilt allerdings auch, dass in Arbeitsbereichen, in denen primär Fähigkeiten eingesetzt werden müssen, die von altersbedingten Abbauprozessen stark betroffen sind, im höheren Lebensalter Leistungsreduktionen wahrscheinlicher werden. Dies betrifft insbesondere Tätigkeiten mit hohen physischen Anforderungen oder hohen Anforderungen an die Sinnesleistungen, sowie Arbeitszusammenhänge, die aufgrund eingeschränkter Handlungs- und Entscheidungsspielräume individuelle Kompensationsstrategien nicht zulassen (vgl. u. a. Wild-Wall et al. 2009; Sonntag 2012). Diese fehlende physische und qualifikatorische Eignung Älterer für bestimmte Anforderungen und Tätigkeiten (,unzureichende Passgenauigkeit") konstituiert sich somit primär als mangelnde Übereinstimmung von Arbeitsanforderungen und verändertem Leistungsvermögen.

In der Literatur wird in diesem Zusammenhang auch auf Arbeitsplätze mit begrenzter Tätigkeitsdauer hingewiesen (Behrens und Elkeles 2008; Kistler et al. 2009), d. h. bei denen eine Beschäftigung bis zur Altersgrenze normalerweise nicht möglich ist. Dabei handelt es sich vorzugsweise um solche, die mit schwerem Heben und Tragen (z. B. in der Bauindustrie, der Pflege), mit besonderen körperlichen Anforderungen (wie z. B. in der Feuerwehr, beim Bau), mit negativen Arbeitsumgebungseinflüssen wie Hitze, Nässe, Lärm, mit einseitigen Zwangshaltungen, mit Schicht- und Nachtarbeit oder mit besonderen Anforderungen an Konzentration, Monotonieresistenz und Daueraufmerksamkeit verbunden sind (z. B. Programmierung, Anlagenkontrolle), sowie um Tätigkeiten mit geringer Autonomie, insbesondere bei eng getakteten Zeitvorgaben (vgl. Brussig und Schwarzkopf 2013).

Eine vermeintlich geringere Arbeitsproduktivität oder auch ein genereller Erfahrungsvorsprung Älterer kann dabei weder aus dem altersspezifischen Leistungswandel noch 
aus den vorgenannten direkten arbeitsbedingten Einflüssen abgeleitet werden. Die These einer mit dem Alter global wachsenden Schere zwischen Produktivität und Lohnkosten unterstellt empirisch unangemessen, dass die Produktionsbedingungen über alle Branchen hinweg gleich und damit die daraus hervorgehenden Leistungseinbußen ebenfalls identisch sind. Zwar lassen sich alternsbezogene Produktivitätsunterschiede von Belegschaften bisher kaum messen, da oft gravierende Selektionsmechanismen und Scheinkorrelationen auftreten (vgl. im Überblick Börsch-Supan et al. 2009; Flüter-Hoffmann und Sporket 2013), die vorliegenden Befunde legen allerdings in Abhängigkeit von Anregungsniveau und Belastungsmerkmalen der dominanten Arbeitsstrukturen eine differentielle Produktivitätsentwicklung im Alter nahe (Veen 2008; Börsch-Supan et al. 2009; Göbel und Zwick 2009). Von daher scheint es notwendig, alterstypische Risikofaktoren und Leistungspotentiale jeweils vor dem Hintergrund von Veränderungen auf den Produktionsund Dienstleistungsmärkten und der spezifischen Humanressourcennutzung im Betrieb zu analysieren. Insbesondere auf Grund der kumulativen Effekte von physischen und psychischen Belastungen über den Erwerbsverlauf kommt dabei einer Längsschnittperspektive eine hohe Bedeutung zu (Naegele und Sporket 2010).

\subsection{Gesundheitliche Risiken}

Nachlassende Gesundheit kann als das zentrale Risiko für die Einschränkung der beruflichen Leistungsfähigkeit von alternden Belegschaften angesehen werden. Den psycho-physischen Belastungen und Anforderungen in der Erwerbsarbeit muss hierbei ein hoher Stellenwert eingeräumt werden. Auch zeigt sich, dass die Bedeutung dieses Ursachenkomplexes keineswegs abnimmt, sondern sich eher Bedeutungsverschiebungen einzelner Faktoren in diesem Komplex abzeichnen.

Das erhöhte Gesundheitsrisiko älterer Arbeitnehmer dokumentiert sich insbesondere darin, dass sie zwar seltener, dafür aber jeweils länger krank sind als jüngere - mit der Folge von mit dem Alter immer längeren Arbeitsunfähigkeitsdauern pro Fall (vgl. u. a. BKK 2010). In zahlreichen Fällen mündet dieses höhere Krankheitsrisiko in gesundheitsbedingter Frühinvalidität: Die Zugangsquoten für den Übergang in eine Erwerbsminderungsrente steigen ab dem 50. Lebensjahr deutlich an (Rehfeld 2006), das durchschnittliche Zugangsalter in eine Erwerbsminderungsrente liegt mit derzeit rd. 50 Jahren nicht zuletzt deshalb relativ hoch (DRV Bund 2011).

Die kumulativ wirkenden Anforderungen und Belastungen der Arbeitsplätze können hier neben biologischen Altersfaktoren als mitentscheidend angesehen werden (vgl. Siegrist und Dragano 2007; Behrens und Elkeles 2008; Bäcker et al. 2009; Kistler 2009). So zeigen etwa Berufs- gruppen mit hohen psycho-physischen Arbeitsanforderungen z. B. im Bau- oder Pflegesektor deutlich höhere Erwerbsminderungsraten als akademisch geprägte Berufe (vgl. u. a. BKK 2010; Meyer et al. 2011). Bemerkenswert ist in jüngster Zeit die steigende Bedeutung von psychischen und psychiatrischen Erkrankungen als Berentungsursache, die auf Belastungsveränderungen am Arbeitsplatz verweisen $-z$. B. durch eine zunehmend knappere Personalbemessung, steigenden Zeitdruck und leistungsorientiertere Entlohnungsformen (vgl. Bäcker et al. 2009; Siegrist et al. 2009; Lenhardt et al. 2010).

Fasst man die vorliegenden Befunde zum Zusammenhang von Altern und Gesundheitszustand zusammen, so lassen sich vor allem folgende drei Gruppen von betrieblichen Risikofaktoren identifizieren, die über den Berufsverlauf, d. h. mit längerer Expositionsdauer, mit einem hohen Gesundheitsrisiko korrelieren und infolgedessen in besonderer Weise die Beschäftigungschancen in alternden Belegschaften beeinträchtigen (vgl. u. a. Naegele 2004; Bäcker et al. 2009; Kistler et al. 2009):

- zu hohe physisch-psychische Anforderungen (z. B. statische Muskelarbeit, hoher Krafteinsatz, Heben und Tragen, hohe Konzentrationsanforderungen, ungünstige Arbeitszeitregelungen);

- stark belastende physikalisch-chemische Arbeitsumgebung (z. B. Lärm, Staub, witterungsbedingte Einflüsse);

- mangelhafte Arbeitsorganisation, neue Formen der Arbeitsteilung und gestiegene Leistungskontrolle (z. B. Mangel an Freiheitsgraden, Zeit- und Termindruck, Verantwortungsdruck, Rollenkonflikte in der Arbeit).

\subsection{Qualifizierungsrisiken}

Die Ergebnisse der Arbeitsmarkt- und Qualifikationsforschung zeigen, dass in den nächsten Dekaden mit einem forcierten Anstieg der Arbeits- und Qualifikationsanforderungen zu rechnen ist, der die Arbeitslandschaft und damit den betrieblichen Arbeitskräftebedarf erheblich verändern wird (vgl. Expertenkommission Finanzierung Lebenslangen Lernens 2004; Vogler-Ludwig und Düll 2013). Im Übergang zu neuen Produktionstechniken und zur Dienstleistungsgesellschaft verändern sich die Branchen- und Tätigkeitsstrukturen, unterliegen formelle Ausbildungsabschlüsse einem schnellen Alterungsprozess und wachsen vor allem die Anforderungen an die (fachübergreifenden) Qualifikationen („Schlüsselqualifikationen“) und die Flexibilität der Beschäftigten (Zika et al. 2012). Auch die Beschäftigten auf einfacheren Arbeitstätigkeiten sind davon nicht ausgenommen und sehen sich einem verstärkten Druck zur Herausbildung von Problemlöse- und Kommunikationskompetenz ausgesetzt (vgl. Baethge et al. 2013). 
Diesen Entwicklungen stehen gegenwärtig noch vorherrschende Qualifizierungsrisiken älterer Arbeitnehmer/innen und eine altersselektive Qualifizierungspraxis im Betrieb gegenüber. Letzteres drückt sich u. a. darin aus, dass 50bis 64-Jährige eine signifikant geringere Weiterbildungswahrscheinlichkeit zeigten (Rosenbladt und Bilger 2008). Im Zeitraum zwischen 1979 und 2010 konnten die 50- bis 64-Jährigen zwar einen deutlichen Anstieg der Beteiligungsrate an Weiterbildung im Allgemeinen von 11 auf $38 \%$ verzeichnen, da diese aber nach Prozentpunkten ähnlich hoch ausfiel wie in der Altersgruppe der 35- bis 49-Jährigen ist der Abstand zwischen beiden Altersgruppen im Wesentlichen gleich geblieben und beträgt derzeit 10 Prozentpunkte (vgl. Gnahs und Rosenbladt 2010, S. 80). Auch an der betrieblichen Weiterbildung im Besonderen nehmen ältere Beschäftigte in deutlich geringerem Maße teil als alle Altersgruppen zusammen. Während die 55- bis 64-Jährigen im Jahr 2010 nur eine Teilnahmequote von $20 \%$ aufweisen konnten, lag die Teilnahmequote insgesamt bei $26 \%$ (vgl. Gnahs und Rosenbladt 2010, S. 82). Die deutlich niedrigeren Teilnahmequoten älterer Arbeitnehmer können sowohl auf betriebliche Hemmnisse bzw. Kosten-Nutzen-Erwägungen als auch auf die niedrigere Erwerbsbeteiligung älterer im Vergleich zu jüngeren Arbeitnehmern/innen zurückgeführt werden (Koller und Plath 2000; Expertenkommission Finanzierung Lebenslanges Lernen 2004).

Neben dieser altersselektiven Qualifizierungspraxis sind alterstypische Risikofaktoren vor dem Hintergrund von Veränderungen auf den Produktions- und Dienstleistungsmärkten und in Bezug auf die geforderten Qualifikationen $\mathrm{zu}$ thematisieren. Folgende Risikofaktoren sind hierbei zu nennen (vgl. Naegele 2004; Frerichs 2007):

- Zum einen können durch eine jahrzehntelange Konzentration der Arbeitstätigkeit auf bestimmte Verfahren, Arbeitsbereiche oder Arbeitsvorgänge betriebsspezifische Einengungen der Qualifikation im Erwerbsverlaufd. h. mit zunehmendem Alter - verursacht werden. Dies kann dazu führen, dass das ursprünglich vorhandene Qualifikationsvermögen z. T. erheblich beeinträchtigt wird (,disuse-Effekt").

- Zum anderen stellen Dequalifizierungsprozesse in Zusammenhang mit der Einführung neuer Technologien und neuer arbeitsorganisatorischer Konzepte ein zentrales Qualifizierungsrisiko für ältere Arbeitnehmer dar. Dequalifizierungen entstehen vor allem dadurch, dass neue Qualifikationen nachgefragt werden, die von älteren Beschäftigten nicht oder noch nicht erbracht werden können, entweder weil sie im Rahmen der schulischen/ beruflichen Ausbildung nicht vermittelt worden sind oder neuartiges Berufswissen erfordern, das erst durch eine umfassende Weiterbildung erworben werden kann.
- Zuletzt ist auf die eingangs bereits erwähnten alterstypischen Verschiebungen innerhalb der kognitiven Leistungsmerkmale zu verweisen. Einer sich mit dem Alter verringernden Geschwindigkeit der Informationsaufnahme und -verarbeitung stehen eine prinzipiell gleichbleibende Lern-, Konzentrations- und Merkfähigkeit gegenüber. Allerdings kann auch die Lernfähigkeit und -bereitschaft auf Grund fehlender betrieblicher Impulse reduziert sein (Baethge-Kinsky 2008).

Das Auftreten und die Bedeutung altersspezifischer Qualifizierungsrisiken muss dabei insgesamt in Abhängigkeit von der jeweiligen Humanressourcennutzung im Betrieb gesehen werden (s. unten), eine rein abstrakt geführte Diskussion über alterstypische Leistungsveränderungen, über alterstypische $\mathrm{Zu}$ - und Abnahmen oder über die Konstanz einzelner Leistungskomponenten ist wenig zielführend.

\subsection{Motivationsrisiken}

Entwicklungsprozesse von Arbeitsmotivation bzw. arbeitsmotivationsrelevanten Einstellungen im Lebensverlauf sind bislang vergleichsweise wenig untersucht worden. Die vorliegenden Forschungsbefunde - obgleich auf unterschiedlichen Motivationskonzepten und Messkonstrukten basierend - deuten allerdings darauf hin, dass sich im höheren Lebensalter die Faktoren für Arbeitsmotivation und -zufriedenheit verändern (vgl. Roßnagel und Hertel 2006; Brinkmann 2009; Becker-Stiftung 2009). Die Ergebnisse lassen sich aber - entgegen gängiger Vorurteile - nicht pauschal als ein Nachlassen der Arbeitsmotivation im Alter interpretieren, sondern deuten auf einen komplexen Motiv- bzw. Einstellungswandel hin.

Arbeits- und organisationspsychologische Untersuchungen zu Arbeitsmotivation, Verhaltensstilen und motivationsrelevanten Orientierungen zeigen, dass ältere Arbeitnehmer insgesamt eine höhere Zufriedenheit mit ihrer Arbeit, ein größeres Engagement und stärkere organisationale Bindung im Vergleich zu jüngeren Arbeitnehmern aufweisen (vgl. im Überblick Grube und Hertel 2008; vgl. auch Becker-Stiftung 2009; Dittrich 2012). Neuere Studien kommen zu dem Ergebnis, dass ältere Mitarbeiter im Gegensatz zu jüngeren tendenziell ein geringeres Interesse an inner- und außerbetrieblicher Mobilität, beruflicher Entfaltung und beruflichen Veränderungen, Aufstieg und finanziellen Gratifikationen haben und in stärkerem Maße Sicherheitsorientierungen (z. B. Arbeitsplatzsicherheit) entwickeln (Brinkmann 2009). Umgekehrt gewinnen etwa der Wunsch nach Anerkennung und Weitergabe des Erfahrungswissens, kooperativen Arbeitszusammenhängen, größeren Handlungsspielräumen in der Arbeit und Partizipation an Entscheidungsprozessen im höheren Alter an Bedeutung (Hertel et al. 2013). 
Die Auswirkungen von betrieblichen Restrukturierungen und Veränderungen in den Arbeitsprozessen auf die Entwicklung der Arbeitsmotivation im Erwerbsverlauf sind ebenfalls differenziert zu betrachten. Sofern betriebliche Umstrukturierungen mit erweiterten Handlungsspielräumen und angereicherten Arbeitsinhalten einhergegangen sind, scheint sich dies positiv auf die weitere Entwicklung der Arbeitsmotivation älterer Beschäftigter auszuwirken (Heisig 2009; Dittrich 2012). Sofern allerdings dadurch die Arbeitsplatzunsicherheit zunimmt und die Stressbelastung steigt, zeigen sich gegenteilige Effekte, die sich dauerhaft negativ auf die Motivation auswirken können (EU-Expertengruppe HIRES 2009; Hentrich und Latniak 2013). Arbeitsbedingt können dann auch motivationale Probleme entstehen, wenn Karriere- und Gratifikationserwartungen enttäuscht werden, Konkurrenzsituationen mit jüngeren Kollegen auftreten, psychische Überforderungen („BurnOut") vorliegen, Erfahrungswissen entwertet wird und Qualifikationen veralten. Auch Reputationsverluste aufgrund der Versetzung an gering angesehene „Schonarbeitsplätze“ bei gesundheitlichen Einschränkungen sind hier als zentrale Verursachungsfaktoren zu nennen (Naegele 2005, Heisig 2009).

Ebenso können durch bestimmte Lohn-Leistungs-Konfigurationen psychophysische Verschleißprozesse sowie Entmutigung und Demotivierung verursacht werden, insbesondere wenn entsprechende Gratifikationsmodelle gesundheitsschädigende Überforderungssituationen forcieren, wie z. B. in Akkord- und Schichtarbeit (BMFSFJ 2006). Dies gilt insbesondere für alterskritische Arbeitsbereiche, die z. B. mit hohen körperlichen oder arbeitszeitlichen Anforderungen verbunden sind: Nachlassende Kräfte erfordern hier von älteren Arbeitnehmern zusätzliche Anstrengungen, um die betrieblichen Leistungsnormen zu erfüllen - sofern diese nicht entsprechend angepasst werden bzw. eine Versetzung an altersadäquate Arbeitsplätze erfolgt (vgl. Kanfer und Ackerman 2004; Knauth et al. 2009).

\subsection{Leistungsfähigkeit alternder Belegschaften und Produktionsregime}

Vor dem Hintergrund des in Deutschland stattfindenden sozio-demografischen und technologischen Wandels in der Erwerbsarbeit sind die Beschäftigungschancen und -risiken älterer Arbeitnehmer über die angeführten einzelnen Risikobereiche hinaus differenziert zu betrachten. Neben eher deskriptiven tätigkeits-, branchen- und betriebsgrößenspezifischen Analysen und der Reflektion genereller Veränderungen in der Arbeitswelt durch Rationalisierungsprozesse und neue Technikkonzepte hat sich hierfür in der Vergangenheit eine Verknüpfung der alternsspezifischen Beschäftigungsrisiken mit einer spezifischen Systematisierung der betrieblichen Arbeitsbedingungen im Sinne von
Produktionsregimen erwiesen (Frerichs 2002). In diesem Erklärungsansatz wird von unterschiedlichen Strategien der Humanressourcennutzung in Unternehmen ausgegangen, die mit spezifischen Belastungssyndromen und Möglichkeiten der Qualifikations- und Motivationsentwicklung einhergehen (vgl. Rosenow und Naschold 1994; vgl. ähnlich auch Nienhüser 2002; Richenhagen 2011). Für die Entwicklung von Altersrisiken und -effekten werden vor allem die Differenzierungsformen horizontaler und vertikaler Arbeitsteilung, die mit den Arbeitsstrukturen verbundenen Lernpotentiale, sowie die Leistungs- und Qualifizierungspolitik als entscheidend angesehen. Unterschieden werden in der bisherigen Diskussion drei Produktionsregime (vgl. im folgenden Rosenow und Naschold 1994; Frerichs 2002):

- das tayloristische Produktionsregime,

- das Produktionsregime der diversifizierten Qualitätsproduktion und

- das Produktionsregime der innovationsorientierten Qualitätsproduktion.

Dem tayloristischen Produktionsregime, das vornehmlich in Betrieben mit Massengüterprodution vorzufinden ist, werden nur geringe Entwicklungs- und Lernpotentiale und eine lebenszeitlich begrenzte Nutzung von Humanressourcen zugeschrieben. Die Beschäftigten werden vor allem unter der Perspektive der Kostenverursachung betrachtet, die Personalfluktuation kann als sehr hoch angesehen werden. Die Arbeitsteilung in diesem Bereich gilt als sehr stark vorangeschritten, es werden vornehmlich gering qualifizierte Arbeitskräfte, insbesondere Frauen und Ausländer, eingesetzt und der Entscheidungs- und Kontrollspielraum ist sehr begrenzt. Die Arbeitstätigkeit ist durch starke körperliche Belastungen mit entsprechenden Verschleißrisiken geprägt. Typische Beispiele bilden hier z. B. die Nahrungs- und Genussmittelindustrie. Auch wenn tayloristisch geprägte Produktions- und Dienstleistungsbereiche insgesamt an Bedeutung verloren haben, kann aufgrund von vielfältigen Beharrungselementen und Rückentwicklungen von einem Verschwinden bzw. einer Reduzierung dieses Beschäftigungssegmentes auf eine zu vernachlässigende Restgröße derzeit noch keine Rede sein (vgl. Bäcker et al. 2010; Zika et al. 2012).

Tayloristische Arbeitsformen gelten seit jeher für alle Arbeitnehmer, insbesondere aber auch für ältere, als mit einem hohen gesundheitlichen und qualifikatorischen Gefährdungspotential behaftet (vgl. u. a. Ulich 2011; Börner et al. 2013). Bezogen auf die Entwicklung der psychophysischen Leistungsfähigkeit wird zwar tendenziell eine Verringerung körperlicher Belastungen durch Automatisierungsprozesse konzediert, es kann aber dennoch ein hohes Belastungspotential als gegeben angesehen werden. Dieses leitet sich zum Teil aus der Arbeitstätigkeit als solcher ab wie z. B. der Persistenz von Arbeitsumgebungsbelastungen 
-, ist aber durchgängig auch durch die produktionsregimespezifische Leistungsabforderung bedingt. Hierzu tragen die Leistungslohnbedingungen ebenso wie die stark begrenzten beruflichen Aufstiegsmöglichkeiten bei. Die Personalentwicklung in zahlreichen Niedriglohnbetrieben ist nach wie vor im Wesentlichen auf eine low-cost-Politik ausgerichtet, systematische Maßnahmen zur Berufslaufbahngestaltung und zur Höherqualifizierung werden nicht in größerem Umfang entwickelt oder angewendet (Morschhäuser 2006). Die niedrigeren Qualifikationsanforderungen führen stattdessen im Berufsverlauf dazu, dass mit zunehmendem Alter die Entwicklungs- und Umsetzungschancen geringer werden (Baethge-Kinsky 2008). In der Konsequenz herrscht in den meisten Betrieben eine hohe Fluktuation vor, die verbliebenen älteren Mitarbeiter stellen meist eine positive Auslese im Sinne eines ,healthy-worker' Effektes dar und können nicht als Beleg für eine im Betrieb grundsätzlich realisierbare Alterserwerbsarbeit gewertet werden (Bäcker et al. 2009).

Im Produktionsregime der diversifizierten Qualitätsproduktion, bei dem die Fertigung von qualitativ hochwertigen Kleinserien oder Einzelprodukten im Vordergrund steht - als typische Beispiele können der (Werkzeug-)Maschinenbau und Teile der Chemie- und Druckindustrie genannt werden - kommt durch relativ lernoffene Arbeitsstrukturen und die große Arbeitseinsatzbreite der Qualifikation der Beschäftigten und ihrer Entwicklung eine ungleich höhere Bedeutung zu (vgl. für den Maschinen- und Anlagenbau z. B. Pfeiffer et al. 2012). Investitionen in das Humankapital werden als ertragssteigernd angesehen. Die Vielseitigkeit der qualifizierten Tätigkeitsanforderungen unterstützt prinzipiell die Anpassung an neu auftretende Anforderungen. Es dominiert die Beschäftigung männlicher Facharbeiter in langfristigen Beschäftigungsverhältnissen.

In der diversifizierten Qualitätsproduktion und -dienstleistung kann im Gegensatz zum tayloristischen System von einer weit höheren Beschäftigungsqualität für alternde Belegschaften ausgegangen werden. Hier haben sich in der Vergangenheit Arbeitsstrukturen herausgebildet, die insbesondere über die Erfahrungsgebundenheit der Tätigkeit und im Zusammenhang mit der hohen Betriebsbindung zu tendenziell günstigeren Lebenslagen geführt haben. Ältere Arbeitnehmer können akkumuliertes Fach- und Beratungswissen einsetzen und erreichen in der innerbetrieblichen Wertschätzung z. T. hohes Ansehen als Experten und Multiplikatoren (Sporket 2011; Weimer 2012). Zum anderen ist es aber auch aus betrieblicher Sicht wichtig, die Humankapitalinvestitionen - die betriebliche Qualifizierung ist kostenintensiv und erfordert eine lange solide Ausbildung - zu amortisieren, so dass ältere Mitarbeiter auch aus diesem Grund eine hohen Integrationsgrad aufweisen können.

Allerdings kann eine auf die angeführten Aspekte gerichtete eindimensionale und undifferenzierte Bewertung der Beschäftigungsbedingungen als altersunkritisch bzw. alternsgerecht nicht aufrechterhalten werden und es ist davon auszugehen, dass in der diversifizierten Qualitätsproduktion die Altersrisiken bisher unterschätzt worden sind. Hervorzuheben sind zum einen die zunehmende Realisierung technikorientierter Rationalisierung und Gestaltungskonzepte in der Arbeit, die die Gefahr einer Erosion des Erwerbs und der tatsächlichen Nutzung von erfahrungsgebundenen Qualifikationen und Kompetenzen mit sich bringt (vgl. hierzu u. a. Moldaschl 2005; Hentrich und Latniak 2013) und zum anderen Outsourcing-Prozesse, die ebenfalls die Lernchancen in der Arbeitstätigkeit beeinträchtigen können (Bergmann 2000).

Zudem muss auf die gestiegenen psychischen Anforderungen in der Arbeitstätigkeit, die sich aus einer zunehmenden Komplexität, Geschwindigkeit und geforderten Genauigkeit der maschinengeführten Prozesse ergeben, hingewiesen werden (vgl. im Überblick Badura et al. 2009; Lenhardt et al. 2010). Gefordert sind damit erhöhte Aufmerksamkeit, Reaktionsschnelligkeit und Abstraktionsvermögen. Dadurch wird zwar zum einen die Fachlichkeit der Arbeit gestärkt, auf der anderen Seite kommt es jedoch zu einer Komplexitätssteigerung der Tätigkeiten, die mit einer Arbeitsverdichtung einhergeht und das Belastungspotential erhöht. So können aufgrund der technisch-sachlichen Gegebenheiten, z. B. durch Schichtarbeit, hohe Arbeitsbelastungen auftreten (Latniak et al. 2010).

Im Produktionsregime der innovationsorientierten Qualitätsproduktion schließlich geht mit einer entwickelten Aufgabenintegration und Arbeitseinsatzflexibilität eine noch sehr viel stärkere qualifikationserweiternde Nutzung der Arbeitskräfte einher. Die Belegschaften sind oft sehr stark jugendzentriert und stellen eine zentrale strategische Ressource für die Realisierung von Wettbewerbsvorteilen dar. Das permanent hohe Anforderungsniveau ist allerdings auch mit erheblichen Verschleißrisiken verbunden. Als typischer Bereich kann die Softwareentwicklung genannt werden.

In der innovationsorientierten Qualitätsproduktion herrscht eine starke Dichotomisierung der Beschäftigungschancen zwischen den verschiedenen Altersgruppen vor. Diese stark beschäftigungsexpansiven Betriebe beispielsweise in der Softwarebranche und andere qualifizierte unternehmensbezogene Dienstleistungen wie z. B. in der Werbebranche (vgl. Pohlmann et al. 2003; GWA 2009) sind in ihrer Personalpolitik vorwiegend jugendzentriert ausgelegt. Dies zeigt sich nicht nur in den Rekrutierungsstrategien, sondern auch in der wenig langfristig ausgelegten Personalentwicklungspolitik, die kaum Möglichkeiten für die Kompensation der hohen psychischen Anforderungen bietet. Beschäftigungschancen für alternde Belegschaften müssen erst systematisch erschlossen werden. Es überwiegen hohe Verschleißrisiken insbesondere qualifikatorischer Art, indem durch den schnellen „Wissensumschlag“ in den 
hoch innovativen Arbeitsgebieten permanent oder in schnell aufeinander folgenden halbjährlichen und jährlichen Rhythmen neues Wissen angeeignet und bestehendes erweitert werden muss bzw. Paradigmenwechsel im Anwendungswissen vollzogen werden müssen (Baethge 2011). Entlastende Momente durch das Einspeisen von Erfahrungsmomenten in die Tätigkeit sind dagegen kaum vorhanden. Zum anderen werden aufgrund des vorherrschenden Kosten- und Zeitdrucks oft keine ausreichenden zeitlichen Freiräume gewährt, um diesen Qualifikationsanforderungen über das unbedingt notwendige Maß hinaus nachzukommen (Frerichs 2002).

Hervorzuheben sind zum anderen die psychischen Belastungen, die im Gegensatz zum tayloristischen System nicht durch ein hohes $\mathrm{Ma} ß$ an direkter Leistungskontrolle generiert, sondern über Zeit- und Sachzwänge transportiert werden (vgl. u. a. Hien 2008). Die Kehrseite der die intrinsischen Motivation fördernden und positiv zu bewertenden Arbeitsanreize und -strukturen liegt in dem hohen Leistungs- und Erfolgsdruck, der sich in z. T. stark ausgeweiteten Arbeitszeiten, Zeitdruck und Stress äußert und über den Erwerbsverlauf erschöpfend wirkt (Gerlmaier et al. 2010). Gleichzeitig gehen damit mangelnde Regenerationsmöglichkeiten in der eingeschränkten Freizeit einher. Die Tätigkeitsanforderungen in den hierarchiearmen und hochflexiblen betrieblichen Organisationen sind durch eine hohe inhaltliche Komplexität gekennzeichnet und bringen einen großen Entscheidungs- und Verantwortungsdruck mit sich, auch wenn sich bestimmte Arbeitsaufgaben z. B. durch programmgesteuerte Abläufe routinisieren lassen.

Ältere - und das können bereits ,junge Alte“ im Betrieb, d. h. 35-Jährige und ältere sein - sind daher in ihrer betrieblichen Stellung gefährdet. Ihre Wertschätzung ist hauptsächlich auf quantitativ relativ begrenzte Leitungs- und Sozialisationsfunktionen beschränkt (vgl. Sporket 2011). Die geschilderten Arbeitsstrukturen führen zu einem erhöhten Ausgliederungsdruck und zu Statusabstufungen - realisiert über nachdrücklich durchgesetzte Annahme von Abfindungen, Versetzungen an unattraktive Arbeitsplätze oder Regionen, größere Betroffenheit von betriebsbedingten Kündigungen in Bereichen, die nicht mehr rentabel erscheinen und über die Abwertung der Leistungsfähigkeit im Betrieb (Frerichs 2002).

Insgesamt bleibt darauf hinzuweisen, dass ein vermuteter starker Unterschied zwischen Produktion und Dienstleistung etwa in dem Sinne, dass im Dienstleistungsbereich unabhängig vom Produktionsregime per se bessere Arbeitsund Beschäftigungsbedingungen für ältere Beschäftigte z. B. aufgrund eines niedrigeren körperlichen Belastungsgrades vorherrschen, nicht verifiziert werden kann (vgl. im Überblick Pohlmann et al. 2003). Zwar sind durchaus unterschiedliche Tätigkeitselemente zwischen Dienstleistung und Produktion festzuhalten $-z$. B. in Form eines größe- ren Personenbezugs und z. T. andersartiger arbeitszeitlicher Rahmenbedingungen - insgesamt bleibt aber bezüglich dieser Untersuchungsebene die produktionsregimetypische Bestimmung der Lebenslagen wesentlich (vgl. zur funktionalen Heterogenität im Dienstleistungssektor etwa Baethge 2011).

Die unternehmens- und personenbezogenen Hilfsdienste mit ihren oft prekären Arbeits- und Beschäftigungsbedingungen - niedrige Löhne, oft noch hohe körperliche Anforderungen, tätigkeitsimmanente psychische Belastungen und mangelnde tarifrechtliche Absicherung, wie z. B. in der Transport- und Logistikbranche oder im Gastgewerbe (vgl. hierzu u. a. Maack et al. 2013, im Überblick Bosch \& Weinkopf 2011) - stellen ein bedeutendes Gegengewicht zur Schaffung alternsförderlicher Beschäftigungsbedingungen dar und weisen eine hohe Strukturähnlichkeit mit den Beschäftigungsbedingungen im tayloristischen Produktionsbereich auf (vgl. Jansen und Müller 2000, beispielhaft zum Bereich der Call-Center Dumke 2008). Im Ergebnis wird in beiden Fällen auf Personalfluktuation gesetzt, es herrscht eine geringe Humanressoureninvestition vor und die Lebenslagen älterer Arbeitnehmer sind mit besonders hohen Risiken behaftet.

Auch die qualifizierten Dienstleister sind wie die diversifizierte Qualitätsproduktion als solche von erhöhten Anforderungen an Leistungsbereitschaft und Flexibilität, sowie von zunehmendem Konkurrenzdenken geprägt. Die stärkere Absatz- und Marktorientierung bei gleichzeitig wachsender Aufgabenverantwortung erhöht auch hier die Belastungspotentiale im Erwerbsverlauf (vgl. für den Bankenbereich DAK 2008; Bludau-Hoffmann 2010). Die hohen arbeitsinhaltlichen Anforderungen werden durch arbeitszeitliche Anforderungen zusätzlich stark erhöht. Infolge des Kostendrucks und z. T. gravierenden Umstrukturierungsprozessen kommt es insgesamt zu einer Arbeitsintensivierung und einer damit einhergehenden steigenden psychischen Belastung (vgl. für den Pflegebereich u. a. Büssing und Glaser 2003; Theobald et al. 2013). Auch im diversifizierten Dienstleistungssektor hat das der betrieblichen Wertschätzung zugrundeliegende Prinzip des Erfahrungswissens durch den Einsatz neuer Technologien und Arbeitsorganisationsformen eine Relativierung erfahren müssen, die seine Bedeutung gegenüber Qualifikationen wie Flexibilität, Kreativität und Innovationsfähigkeit erheblich abgesenkt hat.

\section{Betriebliches Alter(n)smanagement}

Die bestehenden Beschäftigungsrisiken erfordern eine längerfristig angelegte, aktive Förderung von Bedingungen, die eine Arbeit in den mittleren und höheren Lebensjahren auch objektiv ermöglichen. Maßnahmen, die der Unterstützung alternder Arbeitskräfte bzw. der Sicherung ihrer Arbeits- 
und Beschäftigungsfähigkeit dienen, dürfen demnach nicht erst am Ende, sondern müssen während der gesamten Erwerbsbiographie zum Einsatz kommen (vom Alters- zum Alternsmanagement). Trotz unterschiedlicher Schwerpunktsetzungen und Forschungsperspektiven besteht ein weitgehender Konsens dahingehend, dass wirkungsvolle Gestaltungsansätze über Einzelmaßnahmen hinausgehen und ganzheitlich - im Sinne eines Managementsystems angelegt sein müssen.

Die für die Diskussion im deutschsprachigen Raum wichtige Unterscheidung zwischen Alters- und Alternsmanagement verweist hierbei auf Unterschiede in der Ausrichtung der jeweiligen Aktivitäten im Hinblick auf Zielgruppen und Zeithorizonte. „Altersmanagement“ steht demnach für Vorgehensweisen, die in erster Linie an die Gruppe derjenigen älteren Arbeitnehmer adressiert sind, deren Leistungsfähigkeit nicht mehr im Einklang mit den beruflichen Arbeitsanforderungen steht. Es handelt sich somit vorrangig um korrektive und/oder kompensatorische Gestaltungsansätze, deren Ziel die Integration leistungsgewandelter älterer Beschäftigter ist, etwa durch die Versetzung an weniger belastende Arbeitsplätze bzw. Herabsetzung der Arbeitsanforderungen, rehabilitative Maßnahmen oder Anpassungsqualifizierungen.

„Alternsmanagement“ hingegen umfasst eine stärker präventiv orientierte, die gesamte Erwerbsbiographie und damit alle Altersgruppen einbeziehende Prozess-Perspektive. Alternsmanagement berücksichtigt, dass Altern als ein lebenslanger, interindividuell varriierender Entwicklungsund Veränderungsprozess verstanden werden muss, und dass für einen Rückgang berufsrelevanter Leistungspotentiale im höheren Lebensalter nicht allein biologische Abbauprozesse verantwortlich sind, sondern in hohem Maße auch die jeweiligen Arbeits- bzw. Lebensbedingungen. In diesem Sinne weist Alternsmanagement zahlreiche Parallelen und Schnittstellen zu den arbeitswissenschaftlichen Konzepten für eine „Humanisierung der Arbeit“ auf. Eine alternsgerechte Arbeits- und Organisationsgestaltung zeichnet sich dabei in besonderer Weise dadurch aus, dass sie in Ergänzung zu bestehenden Ansätzen zusätzlich den altersgruppenspezifischen Unterschieden und den intraindividuellen, über die Lebensspanne sich vollziehenden Veränderungsprozessen hinsichtlich der Leistungspotentiale und persönlichen Bedürfnisse der Beschäftigten in besonderer Weise Rechnung trägt (z. B. Naegele und Sporket 2010; Schlick et al. 2013). Die zur Verfügung stehenden Maßnahmen sollen demnach mit dieser besonderen Schwerpunktsetzung und Zielorientierung eingesetzt und aufeinander bezogen werden (Ilmarinen 2005).

Die obige Definition verweist zusätzlich auf eine besondere Ressourcen-Perspektive: In der wissenschaftlichen Diskussion der jüngeren Zeit wird verstärkt gefordert, altersbezogene Personalkonzepte nicht allein auf eine per- sonenbezogene Schutz- und Kompensationsfunktion zu reduzieren, sondern zugleich auf die stärkere Berücksichtigung und adäquate Nutzung altersspezifischer Fähigkeiten, Fertigkeiten und Kenntnisse hin auszurichten. Mit einer solchen Ressourcenorientierung kann zum einen der gerontologischen Erkenntnis Rechnung getragen werden, der zufolge Alterungsprozesse nicht nur mit negativen Veränderungen der Leistungsfähigkeit einhergehen, sondern zugleich positive Entwicklungsverläufe beinhalten (Kruse 2008; Heinze et al. 2011). Andererseits wird deutlich gemacht, dass Alter(n)smanagement auch der Verfolgung betriebswirtschaftlichen Zielstellungen dienen kann und muss: Die Bewältigung wirtschaftlicher, technologischer und organisatorischer Herausforderungen wird betrieblicherseits aufgrund veränderter demografischer und rentenpolitischer Rahmenbedingungen zukünftig in zunehmendem Maße mit älteren Belegschaften erfolgen müssen. Dies erfordert eine verstärkte Hinwendung zu ressourcenbasierten Unternehmensstrategien, die vor allem auf die Entfaltung von Arbeitsvermögen statt auf Kostensenkung ausgerichtet sind (vgl. Brödner 2011, S. 192).

Da die angeführten Beschäftigungsrisiken in einem interdependenten Verhältnis zueinander stehen, sich also sowohl positiv als auch negativ wechselseitig beeinflussen können, sollten Alters- und Alternsmanagement-Aktivitäten zudem mehrdimensional angelegt sein, und - mit Blick auf die Förderung von Verbundeffekten - systematisch aufeinander bezogen werden (vgl. hierzu auch Hentrich und Latniak 2013). Die angeführten strukturellen Verursachungsfaktoren sprechen zudem dafür, verhältnisorientierte, an den Arbeitsbedingungen ansetzende Maßnahmen stärker zu gewichten als verhaltensbezogene, auch wenn diese in ihrem Stellenwert nicht unterschätzt werden sollten (vgl. Siegrist et al. 2009).

Im Rahmen eines solchen alter(n)gerechten Personalmanagements sind dabei folgende Handlungsebenen $\mathrm{zu}$ berücksichtigen (Naegele 2005; Bögel und Frerichs 2011; Sporket 2011):

- Arbeits(zeit)gestaltung: Der Gestaltung von Arbeitskontexten kommt im Rahmen von Alter(n)smanagement eine übergeordnete Bedeutung zu. Der Gestaltungsbereich umfasst dabei ein weites Spektrum an unterschiedlichen sowohl präventiv und prospektiven, als auch korrektiv-kompensatorisch ausgerichteten Interventionen. $\mathrm{Zu}$ nennen sind hier Maßnahmen des Arbeitsschutzes und der Arbeitsplatzergonomie (z. B. Verringerung der Arbeitsumgebungbelastung, ergonometrische Richtgrößen bei der räumlichen Gestaltung von Arbeitsplätzen, vgl. Landau et al. 2007), Arbeitsstrukturierungskonzepte (u. a. Veränderung des Zuschnitts von Arbeitsaufgaben und der Arbeitsteilung, vgl. Hinz 2013), sowie Ansätze zur Arbeitszeitgestaltung (z. B. Optimierung 
von Schichtplänen und Pausengestaltung, vgl. Lennings 2013). Zukünftig sind dabei insbesondere lebenszyklisch unterschiedliche Zeitbedürfnisse und -präferenzen in erwerbsbiographischer Dimension zu berücksichtigen, z. B. in Form von Erziehungs- und Pflegeurlauben, Sabbaticals und Weiterbildungszeiten bzw. Lernzeitkonten (Schilling 2008; Flüter-Hoffmann und Sporket 2013). In diesem Zusammenhang kann auch der gleitende Übergang in den Ruhestand bzw. die Altersteilzeit an neuer Attraktivität gewinnen (Fröhler et al. 2013).

- Individuelle Gesundheitsförderung: Als besonders relevant für Maßnahmen der individuellen Gesundheitsförderung, die die Arbeitsgestaltung ergänzen aber nicht ersetzen können, lassen sich vier Schwerpunktbereiche identifizieren (Bögel und Frerichs 2011). Insbesondere sind hier die Förderung gesundheitsschonender Arbeitsausführung mit besonderem Fokus auf der Prävention von Muskel-Skelett-Erkrankungen, sowie Förderung der psychischen Belastbarkeit mit besonderem Fokus auf der Stressbewältigungsfähigkeit zu nennen. Aber auch die generelle Förderung der körperlichen Aktivität, gesunder Ernährungsweisen und Suchtprävention müssen als bedeutsam erachtet werden.

- Qualifizierung alternder Belegschaften: Unabhängig vom Alter muss im Sinne eines lebenslangen Lernens ein kontinuierlicher Erwerb von arbeitsplatzbezogenen wie arbeitsplatzübergreifenden Qualifikationen ermöglicht werden. Dazu sollte u. a. die planmäßige Integration von Qualifizierungsprozessen in die Arbeitsorganisation über lernfördernde Arbeitsprozesse und Arbeitsmittel und die systematische Planung von Lernprozessen über inner- und zwischenbetriebliche Mobilitätsförderung und Arbeitsplatzwechsel betrieben werden (vgl. Frerichs 2007; Baethge-Kinsky 2008). Zum anderen müssen für dequalifizierte ältere Arbeitnehmer besondere, kompensatorische Qualifizierungsansätze entwickelt und methodisch-didaktisch auf diese Gruppe zugeschnitten werden.

- Lernen, Fort- und Weiterbildung: Neben der Etablierung lernförderlicher Arbeitssysteme sind verstärkt Weiterbildungsaktivitäten $\mathrm{zu}$ initiieren, da ein ausschließlich arbeitsintegriertes und informelles Lernen die Gefahr einer betriebsspezifischen Verengung von Qualifikationsprofilen birgt (vgl. Frerichs 2007; Zimmermann 2009). Gemäß des zugrunde gelegten Konzeptes von Alter(n)smanagement ist dabei auch hier vorrangig eine präventive Ausrichtung gefordert, was in diesem Fall bedeutet, dass berufsorientierte Weiterbildungsaktivitäten im Sinne eines lebenslangen Lernprozesses über den gesamten Verlauf der Erwerbsbiografie verteilt sein müssen, und demnach nicht nur jüngere Beschäftigte einbeziehen bzw. reaktiv, d. h. erst beim Auftreten akuter Personaleinsatzprobleme, erfolgen sollten. Hierbei sind insbesondere auch Niedrigqualifizierte einzubeziehen (Baethge et al. 2013).

- Motivationsförderung: Die eingangs referierten Forschungsbefunde zur Entwicklung der Arbeitsmotivation deuten darauf hin, dass monetäre Gratifikationen und künstlich geschaffene Wettbewerbssituationen bei älteren Arbeitnehmern offenbar weniger wirkungsvolle Instrumente zur Motivationssteigerung bilden als bei jüngeren Arbeitnehmern. Für die Gestaltung alter(n) sgerechter Gratifikationsmodelle ergibt sich hieraus die Konsequenz, dass bei der Entwicklung von Leistungsmaßstäben auch die Entwicklung des Leistungsvermögens im Altersverlauf Berücksichtigung finden muss, und Anreizstrukturen, die zu gesundheitsschädlichen Arbeitsweisen an belastungsintensiven Arbeitsplätzen motivieren (z. B. in Form von Akkordlöhnen oder auch Nachtarbeitszuschlägen), vermieden werden. Ansatzpunkte für die Entwicklung lernorientierter Gratifikationsmodelle bieten hier möglicherweise sogenannte Polyvalenzlohnsysteme, die neben dem Normallohnsatz, Leistungsboni, Erschwerniszulagen etc. auch die Qualifikation und innerbetriebliche Einsatzflexibilität von Beschäftigten honorieren (vgl. Ulich 2006).

- Laufbahngestaltung: Betriebliche Laufbahngestaltung ist ebenso wie Arbeitsgestaltung als Querschnittsmaßnahme anzusehen, die sowohl Auswirkungen auf Gesundheitsförderung und Qualifikationserhalt als auch Motivation haben kann, sofern Anforderungen, Anreize und Belastungen im Erwerbsleben zeitlich und inhaltlich adäquat aufeinander folgen und einem frühzeitigen Verschleiß der Leistungsfähigkeit entgegenwirken (vgl. Behrens und Elkeles 2008). Hierbei ist sowohl auf veränderte Zuschnitte von Tätigkeiten, eine horizontale Laufbahngestaltung, Konzepte des job-enrichment als auch auf altersgemischte Arbeitsgruppen abzuheben, um die Chancen für einer länger andauernde Erwerbstätigkeit zu erhöhen (vgl. beispielhaft Morschhäuser 2006; Bertelsmann Stiftung \& BDA 2008; Frerichs et al. 2012).

Die genannten Gestaltungsansätze und -spielräume sind wiederum als abhängig von den spezifischen betrieblichen Produktionsregimen zu sehen. Im tayloristischen System kommt auf Grund der low-cost-politik weiterhin der Bekämpfung von elementaren Gesundheitsrisiken und der Schaffung grundlegender Qualifikationsstrukturen in den Unternehmen, z. B. durch Einführung teilautonomer Arbeitsgruppen (vgl. hierzu u. a. Ulich 2011; Hentrich und Latniak 2013), eine hohe Bedeutung zu. In der diversifizierten Qualitätsproduktion und -dienstleistung sind die Gestaltungsspielräume zur Verbesserung der Lebenslage älterer Arbeitnehmer höher zu bewerten, müssen aber auch hier erst aktiv erschlossen werden und betriebliche Interessenvertretungen eigene Problemlösekompetenzen entwickeln. 
Prinzipiell geht es dabei stärker als im tayloristischen System um die Sicherung bzw. die Wiederherstellung altersintegrativer und erfahrungsgebundener Arbeitsprozesse. Eine bewusste Reintegration von produktivitätsfördernden erfahrungsgebundenen Tätigkeitselementen in den konkreten Arbeitsprozess muss sehr viel stärker durchgesetzt und bewusst gestaltet werden (vgl. Böhle 2009).

In der innovationsorientierten Qualitätsproduktion und -dienstleistung kann kaum auf ,altbewährte' Handlungsrezepte zurückgegriffen oder der Erfahrungsgehalt einzelner Tätigkeiten gefördert werden. Auch die Einwirkung auf physische Belastungsfaktoren spielt eine eher untergeordnete Rolle. Vielmehr geht es darum, dass auf der betrieblichen Ebene die Gestaltung von Arbeitsorganisation und Personalpolitik integriert wird, um zu verhindern, dass typische Sackgassenbereiche für ältere Arbeitnehmer entstehen. Insbesondere in den Handlungsfeldern Arbeitszeitgestaltung (vgl. Gerlmaier et al. 2010) und Geschäftsfeldpolitik (Frerichs 2002) bietet sich ein hohes Gestaltungspotential, da bestehende arbeitsorganisatorische und -inhaltliche Freiräume genutzt werden können.

\section{Akteursbezogene Perspektiven der Alterserwerbsarbeit}

Es spricht vieles dafür, dass künftige Belegschaften von Betrieben und Verwaltungen insgesamt älter sein werden. Aufgrund der aufgeführten Beschäftigungsrisiken und des jahrzehntelangen Trends zur Frühverrentung fehlt es derzeit in der überwiegenden Zahl der Betriebe und Verwaltungen aber an Erfahrungen und positiven Ansätzen für ein alternsbezogenes Personalmanagement. Innovative Handlungsansätze, die den älteren Beschäftigten einen dauerhaften Verbleib im Erwerbsleben ermöglichen, sind zwar auf Grund einer Vielzahl an wissenschaftlichen Analysen, Handlungsleitfäden und Übersichten vorbildlicher Praxis (vgl. z. B. Morschhäuser 2006; Naegele und Walker 2006; Sporket 2011; im Überblick Bögel 2013) seit längerem bekannt. Aus quantitativer Sicht - und trotz einer breitflächigen Projektförderung (vgl. Pfeiffer und Richter 2011) - stellt sich aber die breitenwirksame Umsetzung von betrieblichen Aktivitäten des Alternsmanagements bisher als unzureichend dar (Bellmann et al. 2007; Stettes 2012; Leber et al. 2013; vgl. im Überblick Bögel 2013).

Vieles spricht in diesem Zusammenhang dafür, dass Ansätze der Personalentwicklung erst dann die nötige Breitenwirkung und Nachhaltigkeit entfalten, wenn sie in ein umfassendes personalstrategisches Konzept integriert werden. Sowohl die Durchführung eines systematischen Altersstruktur- und Personalentwicklungs-Monitoring als auch die Umsetzung der vorgestellten Schwerpunktinterventionen sollten daher sukzessive in eine gesamtbetriebliche Her- angehensweise im Sinne eines systematischen und mehrdimensionalen Alter(n)smanagement eingebunden werden. Hierdurch sollte auch gewährleistet werden, dass die einzelnen Handlungs- und Interventionsstrategien im Betrieb nicht isoliert betrachtet werden (also z. B. nur Gesundheitsförderung oder nur Kompetenzentwicklung), sondern aufeinander bezogene Teilaspekte einer gesamtbetrieblichen Sichtweise und Strategie abbilden. Inwieweit hier neu geschaffene Auditierungssysteme (vgl. im Überblick BAuA 2011) Unternehmen bei der Analyse von Handlungsbedarfen und der Entwicklung von Maßnahmen für wirksame Personalarbeit im demografischen Wandel wirksam unterstützen können, muss bislang aber noch offen bleiben.

Neuere Untersuchungen zeigen zudem, dass die Einführung von Alter(n)smanagement als mikropolitischer Prozess $\mathrm{zu}$ begreifen ist, der die Interessen und Interessensgegensätze der betrieblichen Akteure stärker in den Blick nehmen muss (Sporket 2011; Bögel 2013) bzw. ihre aktivierende Rolle bewusst anerkennen und fördern muss (Naegele und Walker 2006). In diesem Zusammenhang erscheint u. a. eine stärkere tarifpolitische Flankierung zur Durchsetzung einer arbeitsgestalterischen Perspektive für alternde Belegschaften notwendig zu sein. Bisher vorliegende Evaluationen verweisen darauf, dass hierdurch nicht nur die gegenwärtigen und zukünftigen betriebsdemografischen Herausforderungen stärker in das Blickfeld von Betriebsräten, Personalleitern und Geschäftsführung geraten, sondern auch konkrete Handlungsanforderungen und -anreize damit verbunden sind (Latniak et al. 2010; Katenkamp et al. 2012; Tullius et al. 2012). Allerdings muss berücksichtigt werden, dass auf Grund der stagnierenden bzw. zurückgehenden tarifpolitischen Bindung diese längst nicht für alle Betriebe umzusetzen sind. Zudem zeigt sich, dass die arbeitsgestalterische Perspektive nicht von leistungs- und entgeltpolitischen Problemstellungen entkoppelt werden kann.

Derzeit fehlen zudem weitergehende flankierende Maßnahmen in der staatlichen Arbeitsmarkt-, Arbeitszeit- und Sozialpolitik. Auch wenn weiterhin von einer primären Verantwortungszuweisung an die Unternehmen auszugehen ist, ist eine integrierte Strategie von betrieblichen und staatlichen Regelungsfeldern gefordert und eine sehr viel stärkere Verbetrieblichung von Maßnahmen und Instrumenten der aktiven Arbeitsmarktpolitik (vgl. Frerichs 2009). Dies betrifft vor allem Maßnahmen der Gesundheitsförderung und Rehabilitation, von Qualifizierung und Weiterbildung und auch der Förderung von Arbeitszeitflexibilisierung. Konkrete Maßnahmen, die betriebliche Strategien zur Förderung der Alterserwerbsarbeit unterstützen, sind auf den unterschiedlichsten Feldern anzusiedeln. So ist eine stärkere Verankerung und Implementierung von „zweiten Weiterbildungphasen " im Betrieb durch Struktur- und Fördermaßnahmen erforderlich. Sozialrechtlich muss eine stärkere Absicherung flexibler Arbeitszeitmodelle - z. B. bei 
der Vereinbarkeit von Erwerbstätigkeit und Pflege - ebenso in Betracht gezogen werden wie stärkere, auch finanzielle Anreize für die Betriebe, die Erwerbsunfähigkeit von Beschäftigten zu vermeiden.

\section{Literatur}

BA (Bundesagentur für Arbeit): Ältere am Arbeitsmarkt. BA, Nürnberg (2012)

Bäcker, G.: Erwerbsminderungsrenten - Strukturen, Trends und aktuelle Probleme. Altersübergangs-Report Nr. 2012-03. IAQ, Duisburg (2012)

Bäcker, G., Brussig, M., Jansen, A., Knuth, M., Nordhause-Janz, J.: Ältere Arbeitnehmer. Erwerbstätigkeit und soziale Sicherheit im Alter. VS Verlag für Sozialwissenschaften, Wiesbaden (2009)

Bäcker, G., Kistler, E., Trischler, F.: Rente mit 67? Zu wenig Arbeitsplätze und zu wenig gute Arbeit für ein Arbeiten bis 67. Vierter Monitoring Bericht des Netzwerks für eine gerechte Rente. DGB, Berlin (2010)

Badura, B., Schröder, H., Klose, J., Macco, K. (Hrsg.): Fehlzeitenreport 2009. Arbeit und Psyche: Belastungen reduzieren - Wohlbefinden fördern. Springer, Berlin (2009)

Baethge, M.: Qualifikation, Kompetenzentwicklung und Professionalisierung im Dienstleistungssektor. WSI-Mitteilungen. 9, 447-445 (2011)

Baethge, M., Severing, E., Weiß, R.: Handlungsstrategien für die berufliche Weiterbildung. Bielefeld, Bertelsmann (2013)

Baethge-Kinsky, V.: Lebenslanges Lernen für alternde Belegschaften. In: Bogedan, C., Müller-Schoell, T., Ziegler, A. (Hrsg.) Demografischer Wandel als Chance, S. 163-172. VSA, Hamburg (2008)

BAuA (Bundesanstalt für Arbeitsschutz und Arbeitsmedizin) (Hrsg.): Qualitätssicherung in der Demografieberatung. Bertelsmann, Bielefeld (2011)

Becker Stiftung: Kognition, Motivation und Lernen älterer Arbeitnehmer - neueste Erkenntnisse für die Arbeitswelt von morgen. Dokumentation der Tagung am 18. und 19. September 2008 in Bonn. Köln (2009)

Behrens, J., Elkeles, Th.: Die Renaissance des Berufs als Mobilitätschance. Horizontale und vertikale überbetriebliche Wechsel als (häufig noch misslingende) Bewältigung gesundheitlich begrenzter Tätigkeitsdauer. In: Hien, W., Bödeker, W. (Hrsg.) Frühberentung als Folge gesundheitsgefährdender Arbeitsbedingungen? S. $61 \mathrm{ff}$. NW Verlag, Bremerhaven (2008)

Bellmann, L., Kistler, E., Wahse, J.: Demografischer Wandel - Betriebe müssen sich auf alternde Belegschaften einstellen. IAB-Kurzbericht 21/2007, Nürnberg (2007)

Bergmann, B. Kompetenzentwicklung im Arbeitsprozess. Z. Arbeitswiss. 54, 139-144 (2000)

Bertelsmann Stiftung \& BDA: Demographiebewusstes Personalmanagement. Strategie und Beispiele für die betriebliche Praxis. Bertelsmann, Gütersloh (2008)

BKK Bundesverband: BKK Gesundheitsreport 2010. Gesundheit in einer älter werdenden Gesellschaft. Eigenverlag, Essen (2010)

Bludau-Hoffmann, H., Eberle, R., Holz-Skibinski, K., Spadzinski, U.: Finanzmarktkrise und Verkaufsdruck im Finanzdienstleistungssektor. WSI-Mitteilungen. 7, 380-381 (2010)

BMFSFJ (Bundesministerium für Familie, Senioren, Frauen und Jugend): Fünfter Bericht zur Lage der älteren Generation in der Bundesrepublik Deutschland: Potenziale des Alters in Wirtschaft und Gesellschaft. BMFSFJ, Berlin (2006)

Bögel, J. Die Einführung von betrieblichem Alters- und Alternsmanagement als mikropolitischer Prozess. Kovac, Hamburg (2013)
Bögel, J., Frerichs, F.: Betriebliches Alters- und Alternsmanagement. Books on Demand, Nordersted (2011)

Böhle, F.: Erfahrungswissen - Erfahren durch objektivierendes und subjektivierendes Handeln. In: Bolder, A., Dobischat, R. (Hrsg.) Eigen-Sinn und Widerstand. Kritische Beiträge zum Kompetenzentwicklungsdiskurs, S. 70-88. VS Verlag für Sozialwissenschaften, Wiesbaden (2009)

Börner, K., Scherr, C., Leitner-Mai, B., Spanner-Ulm, B.: Field study of age-critical assembly processes in the automotive industry. In: Schlick, C.M., Frieling, E., Wegge, J. (Hrsg.) Age-differentiated work-systems, S. 252-277. Springer, Wiesbaden (2013)

Börsch-Supan, A., Erlinghagen, M., Jürges, H., Hank, K., Wagner, G.G.: Produktivität, Wettbewerbsfähigkeit und Humanvermögen in alternden Gesellschaften. In: Börsch-Supan, A., Erlinghagen, M., Hank, K., Jürges, H., Wagner, G.G. (Hrsg.) Produktivität in alternden Gesellschaften, S. 9-20. Wissenschaftliche, Verlagsgesellschaft Stuttgart, Stuttgart (2009)

Bosch, G., Weinkopf, C.: Arbeitsverhältnisse im Dienstleistungssektor. WSI-Mitteilungen. 9, 439-446 (2011)

Brinkmann, R.: Berufsbezogene Leistungsmotivation älterer Arbeitnehmer. Logos, Berlin (2009)

Brödner, P.: Innovationen erfordern förderliche Institutionen. In: Jeschke, S., Isenhardt, I., Hees, F., Trantow, S. (Hrsg.) Enabling Innovation. Innovationsfähigkeit - deutsche und internationale Perspektiven, S. 188-193. Springer, Berlin (2011)

Brussig, M., Schwarzkopf, M.: Altersübergänge in der Bauwirtschaft. Prekarisierung vermeiden - Erwerbsbeteiligung stärken. Hans Böckler Stiftung, Düsseldorf (2013)

Büssing, A., Glaser, J.: Arbeitsbelastungen, Burnout und Interaktionsstress im Zuge der Reorganisation des Pflegesystems. In: Büssing, A., Glaser, J. (Hrsg.) Dienstleistungsqualität und Qualität des Arbeitsleben im Krankenhaus, S. 101-129. Hogreve, Göttingen (2003)

DAK (Deutsche Angestellten Krankenkasse): Gesundheitsbilanz Kreditgewerbe. Arbeitsbedingungen und Krankenstand in Banken und Finanzinstituten. Hamburg (2008)

Dittrich, T.: Arbeitsmotivation älterer Mitarbeiter. Eine empirische Untersuchung zur Bedeutung von Arbeitsbedingungen und Motiven. disserta, Hamburg (2012)

DRV Bund: Rentenversicherung in Zeitreihen. Ausgabe 2011. DRV Bund, Berlin (2011)

Dumke, A.: Stress im Call-Center. Eine stressbezogene Arbeitsanalyse. VDM, Saarrbrücken (2008)

EU-Expertengruppe HIRES: Gesundheit und Restrukturierung. Innovative Ansätze und Politikempfehlungen. Hampp, Mering (2009)

Expertenkommission Finanzierung Lebenslangen Lernens: Finanzierung Lebenslangen Lernens - der Weg in die Zukunft. Schlussbericht. Bertelsmann, Bielefeld (2004)

Flüter-Hoffmann, C., Sporket, M.: Arbeit und Beschäftigung im demografischen Wandel - Konsequenzen für das strategische Personalmanagement. In: Hüther, M., Naegele, G. (Hrsg.) Demografiepolitik. Herausforderungen und Handlungsfelder, S. 200221. Springer VS, Wiesbaden (2013)

Frerichs, F.: Zur betrieblichen Beschäftigungssituation älterer Arbeitnehmer - eine lebenslagen- und produktionsregimespezifische Problemanalyse. In: Behrend, C. (Hrsg.) Chancen für die Erwerbsarbeit im Alter S. 47-62. Leske + Budrich, Opladen (2002)

Frerichs, F.: Weiterbildung und Personalentwicklung 40plus: Eine praxisorientierte Strukturanalyse. In: Länge, T.W., Menke, B. (Hrsg.) Generation 40plus. Demografischer Wandel und Anforderungen an die Arbeitswelt, S. 67-104. Bertelsmann, Bielefeld (2007)

Frerichs, F.: Demografischer Wandel und Arbeitsmarktpolitik für ältere Arbeitnehmer/innen: Das Vereinigte Königreich, Japan und Deutschland im Wohlfahrtsstaatsvergleich. Dortmunder Beiträge zur Sozial- und Gesellschaftspolitik, Bd. 59. LIT Verlag, Münster (2009) 
Frerichs, F., Lindley, R., Aleksandrowicz, P., Baldauf, B., Galloway, S.: Active ageing in organisations: a case study approach. Int. J. Manpow. 33(6), 666-684 (2012)

Fröhler, N., Fehmel, T., Klammer U.: Flexibel in Rente. Gesetzliche, tarifliche und betriebliche Perspektiven. edition sigma, Berlin (2013)

Fuchs, J., Söhnlein, D., Weber, B.: Projektion des Arbeitskräfteangebots bis 2050. Rückgang und Alterung sind nicht her aufzuhalten. IAB-Kurzbericht 16/2011. Nürnberg, IAB (2011)

Gerlmaier, A., Kümmerling, A., Latniak, E.: Gesund altern in HighTech-Branchen? Im Spannungsfeld von Innovation und Intensivierung. IAQ-Report 2010-4, VSA, Duisburg, (2010)

Gnahs, D., Rosenbladt, B.v.: Weiterbildung Älterer. In: Rosenbladt, B.v., Bilger, F. (Hrsg.) Weiterbildungsbeteiligung 2010, S. 80-85. Bertelsmann, Bielefeld (2010)

Göbel, C., Zwick, T.: Age and productivity - Evidence from Linked Employer Employee Data. ZEW Discussion Paper 09-020. Zentrum für Europäische Wirtschaftsforschung, Mannheim (2009)

Grube, A., Hertel, G.: Altersbedingte Unterschiede in Arbeitsmotivation, Arbeitszufriedenheit und emotionalem Erleben während der Arbeit. In: Wegge, J., Frieling, E., Schmidt, K.-H. (Hrsg.) Alter und Arbeit. Sonderheft der Wirtschaftspsychologie, 10, S. 18-29. (2008)

GWA (Gesamtverband Kommunikationsagenturen): GWA Human Resources Management Studie. GWA, Frankfurt a. M., (2009)

Hacker, W.: Leistungs- und Lernfähigkeit älterer Menschen. In: Cranach, M.v., Schneider, H.-D., Winkler, R., Ulich, E. (Hrsg.) Ältere Menschen im Unternehmen. Chancen, Risiken, Modelle, S. 163172. Huber, Bern (2004)

Heinze, R.G., Naegele, G., Schneiders, K.: Wirtschaftliche Potentiale des Alters. Kohlhammer, Stuttgart (2011)

Heisig, U.: Wandel von Arbeitsperspektiven zwischen den Generationen. In: Richter,G. (Hrsg.) Generationen gemeinsam im Betrieb S. 35-56. Bertelsmann, Bielefeld (2009)

Hentrich, J., Latniak, E.: Rationalisierungsstrategien im demographischen Wandel. Springer, Wiesbaden (2013)

Hertel, G., Thielgen, M., Rauschenbach, C., Grube, A., Stamov-Roßnagel, C., Krumm, S.: Age differences in motivation and stress at work. In: Schlick, C.M., Frieling, E., Wegge, J. (Hrsg.) Age-differentiated work-systems, S. 119-147. Springer, Wiesbaden (2013)

Hien, W.: Irgendwann geht es nicht mehr. Älterwerden und Gesundheit im IT-Beruf. VSA, Hamburg (2008)

Hinz, A: Arbeitsorganisation und Arbeitsgestaltung - zentrale Handlungsfelder für ein demografiefestes Unternehmen. In: Hentrich, J., Latniak, E. (Hrsg.) Rationalisierungsstrategien im demographischen Wandel, S. 181-198. Springer, Wiesbaden (2013)

Ilmarinen, J.: Towards a longer worklife. Ageing and the quality of worklife in the European Union. Gummerus, Helsinki (2005)

Jansen, R., Müller, R.: Arbeitsbelastungen und Gesundheit älterer Arbeitnehmer im Dienstleistungsbereich. Z. Gerontol. Geriatr. 33(4), S. 256-261 (2000)

Kanfer, R., Ackerman, P.L.: Aging, adult development and work motivation. Acad. Manag. Rev. 29(3), 440-458 (2004)

Katenkamp, O., Martens, H., Georg, A., Naegele, G., Sporket, M.: Nicht zum alten Eisen! Die Praxis des Demographie-Tarifvertrages in der Eisen- und Stahlindustrie. Edition Sigma, Berlin (2012)

Kistler, E.: Alternsgerechte Erwerbsarbeit. En Überblick über den Stand von Wissenschaft und Praxis. Böckler Forschungsmonitoring Nr. 7. Hans-Böckler-Stiftung, Düsseldorf (2009)

Kistler, E., Trischler, F., Bäcker, G.: Rente mit 67- für viele Beschäftigte unerreichbar. Vierter Monitoring Bericht des Netzwerks für eine gerechte Rente. DGB, Berlin (2009)

Knauth, P., Karl, D., Elmerich, K.: Lebensarbeitszeitmodelle. Chancen und Risiken für das Unternehmen und die Mitarbeiter. Universitätsverlag, Karlsruhe (2009)
Koller, B., Plath, H.-E.: Qualifikation und Qualifizierung älterer Arbeitnehmer. Mitteilungen aus der Arbeitsmarkt- und Berufsforschung. 33, 112-125 (2000)

Kruse, A. (Hrsg.): Weiterbildung in der zweiten Lebenshälfte. Bertelsmann, Bielefeld (2008)

Landau, K., Weißert-Horn, M., Rademacher, H., Brauchler, R., Bruder, R., Sinn-Behrendt, A.: Altersmanagement als betriebliche Herausforderung. Ergonomia, Stuttgart (2007)

Latniak, E, Voss-Dahm, D., Elsholz, U., Gottwald, M., Gerisch, S.: Umsetzung demografiefester Personalpolitik in der chemischen Industrie. Hans-Böckler-Stiftung, Düsseldorf (2010)

Leber, U., Stegmaier, J., Tisch, A.: Altersspezifische Personalpolitik. Wie Betriebe auf die Alterung ihrer Belegschaften reagieren. IABKurzbericht 13/2013. IAB, Nürnberg (2013)

Lehr, U.: Psychologie des Alterns. Quelle \& Meyer, Wiebelsheim (2007)

Lenhardt, U., Ertel, M., Morschhäuser, M.: Psychische Arbeitsbelastungen in Deutschland: Schwerpunkte, Trends - betriebliche Umgangsweisen. WSI-Mitteilungen. 7, 335-341 (2010)

Lennings, F.: Arbeitszeit- und Schichtplangestaltung. In Hentrich, J., Latniak, E. (Hrsg.) Rationalisierungsstrategien im demographischen Wandel, S. 199-226. Springer, Wiesbaden (2013)

Maack, K., Haves, J., Homann, B., Schmid, K.: Die Zukunft des Gastgewerbes. Hans Böckler Stiftung, Düsseldorf (2013)

Meyer, M., Stallauke, M., Weirauch, H.: Krankheitsbedingte Fehlzeiten in der deutschen Wirtschaft im Jahr 2010. In: Badura, B., Ducki, A., Schröder, H., Klose, J., Macco, K. (Hrsg.) Fehlzeitenreport 2011. Führung und Gesundheit, S. 223-268. Springer, Berlin (2011)

Moldaschl, M. (Hrsg.): Immaterielle Ressourcen. Hampp, München (2005)

Morschhäuser, M.: Reife Leistung. Personal- und Qualifizierungspolitik für die künftige Altersstruktur. Edition Sigma, Berlin (2006)

Mümken, S., Brussig, M.: Alterserwerbsbeteiligung in Europa. Deutschland im internationalen Vergleich. Altersübergangs-Report Nr. 2012-01. IAQ, Duisburg (2012)

Naegele, G.: Zwischen Arbeit und Rente. Gesellschaftliche Chancen und Risiken älterer Arbeitnehmer. Maro, Augsburg (2004)

Naegele, G.: Nachhaltige Arbeits- und Erwerbsfähigkeit für ältere Arbeitnehmer. WSI-Mitteilungen, 4, 214-219 (2005)

Naegele, G., Walker, A.: A guide to good practice in age-management. European Foundation, Luxemburg (2006)

Naegele, G., Sporket, M.: Perspektiven einer lebenslaufbezogenen Ältere-Arbeitnehmer-Politik. In: Naegele, G. (Hrsg.) Soziale Lebenslaufpolitik, S. 449-474. VS Verlag, Wiesbaden (2010)

Nienhüser, W.: Alternde Belegschaften - betriebliche Ressource oder Belastung? Betriebliche Probleme und Problemhandhabungen in unterschiedlichen Human-Resource-Management-Systemen. In: Behrend, C. (Hrsg.): Chancen für die Erwerbsarbeit im Alter - Betriebliche Personalpolitik und ältere Erwerbstätige, S. 63-85. Opladen: Leske + Budrich (2002)

Pfeiffer, I., Richter, G.: Zwischenbilanz öffentlich geförderter Demografieprojekte. In: BAuA (Hrsg.) Qualitätssicherung in der Demografieberatung, S. 181-194. Bertelsmann, Bielefeld (2011)

Pfeiffer, S., Schütt, P., Wühr, D.: Smarte Innovation. Ergebnisse und neue Ansätze im Maschinen- und Anlagenbau. Springer VS, Berlin (2012)

Pohlmann, M., Sauer, D., Trautwein-Kalms, G. (Hrsg.): Dienstleistungsarbeit:auf dem Boden der Tatsachen - Befunde aus Handel, Industrie, Medien und IT-Branche. Edio Sigma, Berlin (2003)

Rehfeld, U.: Gesundheitsbedingte Frühverrentung. Gesundheitsberichterstattung des Bundes, Heft 30. Robert-Koch-Institut, Berlin (2006) 
Richenhagen, G.: Demografischer Wandel in der Arbeitswelt: Ein internationaler Vergleich in Hinblick auf Arbeits- und Beschäftigungsfähigkeit. In: Schott, T., Hornberg, C. (Hrsg.) Die Gesellschaft und ihre Gesundheit, S. 367-384. VS Verlag für Sozialwissenschaften, Wiesbaden (2011)

Rosenbladt, B.v., Bilger, F.: Weiterbildungsverhalten in Deutschland, Bd. 1: Berichtssystem Weiterbildung und Adult Education Survey 2007. Bertelsmann, Bielefeld (2008)

Rosenow, J., Naschold, F.: Die Regulierung von Altersgrenzen. Strategien und die Politik des Staates. Berlin (1994)

Roßnagel, C., Hertel, G.: Altersbedingte Unterschiede in Inhalten und im Zustandekommen von Arbeitsmotivation und Arbeitszufriedenheit. Z. Arbeitswiss. 60(3), 181-186 (2006)

Schilling, G.: Alternsgerechte Arbeitszeitgestaltung. In: Lorenz, F., Schneider, G. (Hrsg.) Alternsgerechtes Arbeiten, S. 89-124. VSA, Hamburg (2008)

Schlick, C.M., Frieling, E., Wegge, J. (Hrsg.): Age-Differentiated Work-Systems. Springer, Wiesbaden (2013)

Siegrist, J., Dragano, N.: Rente mit 67- Problem und Herausforderungen aus gesundheitswissenschaftlicher Sicht. HBS-Arbeitspapier Nr. 147. HBS, Düsseldorf (2007)

Siegrist, J., Dragano, N., Wahrendorf, M.: Arbeitsbelastungen und psychische Gesundheit bei älteren Erwerbstätigen: die Bedeutung struktureller Intervention. In: Badura, B., Schröder, H., Klose, J., Macco, K. (Hrsg.) Fehlzeitenreport 2009. Arbeit und Psyche: Belastungen reduzieren - Wohlbefinden fördern, S. 167-173. Springer, Berlin (2009)

Sonntag, K.: Arbeitsgestaltung für ältere Beschäftigte. In: Wahl, H.W., Tesch-Römer, C., Ziegelmann, P. (Hrsg.) Angewandte Gerontologie, S. 486-491. Kohlhammer, Stuttgart (2012)

Sporket, M.: Organisationen im demografischen Wandel. Alternsmanagement in der betrieblichen Praxis. VS Verlag für Sozialwissenschaften, Wiesbaden (2011)

Stettes, O.: Altersbilder in deutschen Industrieunternehmen und Personalpolitik für ältere Beschäftigte. In: Berner, F., Rossow, J., Schwitzer, K.-P. (Hrsg.) Altersbilder in der Wirtschaft, im Gesundheitswesen und der pflegerischen Versorgung (S. 25-42). Expertisen zum 6. Altenbericht der Bundesregierung, Bd. 2. VS Verlag für Sozialwissenschaften, Wiesbaden (2012)

Theobald, H., Szebehely, M., Preuss, M.: Arbeitsbedingungen in der Altenpflege. Die Kontinuität der Berufsverläufe - ein deutschschwedischer Vergleich. Edition Sigma, Berlin (2013)

Tullius, K., Freidank, J., Grabbe, J., Kädtler, J., Schröder, W.: Perspektiven alter(n)sgerechter Betriebs- und Tarifpolitik. WSI-Mitteilungen. 2, 113-122 (2012)
Ulich, E.: Lern- und Entwicklungspotentiale in der Arbeit - Beiträge der Arbeits- und Organisationspsychologie. In: Sonntag, Kh. (Hrsg.) Personalentwicklung in Organisationen, S. 138-176. Hogrefe, Göttingen (2006)

Ulich, E.: Arbeitspsychologie, 7. Aufl. Schäffer-Poeschel, Stuttgart (2011)

Veen, St.: Demographischer Wandel, alternde Belegschaften und Betriebsproduktivität. Rainer Hampp Verlag, Mering (2008)

Vogler-ludwig, K., Düll, N.: Arbeitsmarkt 2030. Eine strategische Vorausschau aud Demografie, Beschäftigung und Bildung in Deutschland. Bertelsmann, Bielefeld (2013)

Wanger, S.: Erwerbstätigkeit, Arbeitszeit und Arbeitsvolumen nach Geschlecht und Altersgruppen. IAB-Forschungsbericht Nr. 2. IAB, Nürnberg (2006)

Weimer, S.: Personalentwicklung und Innovation in Unternehmen des Maschinenbaus. In: Pfeiffer, S., Schütt, P., Wühr, D. (Hrsg.) Smarte Innovation. Ergebnisse und neue Ansätze im Maschinen- und Anlagenbau, S. 183-200. Springer VS, Berlin (2012)

Wild-Wall, N., Gajewski, P., Falkenstein, M.: Kognitive Leistungfähigkeit älterer Arbeitnehmer. Zeitschrift für Gerontologie und Geriatrie, 4, S. 299-304 (2009)

Zika, G., Helmrich, R., Kalinowski, M., Wolter, M.I., Hummel, M., Maier, T., Hänisch, C., Drosdowski, T.: Qualifikations- und Berufsfeldprojektionen bis 2030. IAB-Kurzbericht 18. IAB, Nürnberg (2012)

Zimmermann, H.: Kompetenzentwicklung durch Erfahrungstransfer. Berufsbildung in Wissenschaft und Praxis. 34, 26-30 (2005)

Zimmermann, H.: Weiterbildung im späteren Erwerbsleben. Empirische Befunde und Gestaltungsvorschläge. Bertelsmann, Bielefeld (2009)

Frerich Frerichs, hat Soziologie und Psychologie an der FU Berlin studiert und war anschließend als wissenschaftlicher Mitarbeiter (1991 - 1999) und wissenschaftlicher Geschäftsführer (2000 - 2006) am Institut für Gerontologie an der TU Dortmund tätig. Seit 2006 ist er Professor für Altern und Arbeit am Institut für Gerontologie der Universität Vechta. Seine Arbeits- und Forschungsschwerpunkte umfassen die Bereiche Arbeitsmarkt und Altersstrukturwandel, Betriebe im demografischen Wandel und Arbeit und Lebenslagen im Erwerbsverlauf. 